Association for Information Systems

AIS Electronic Library (AISeL)

Wirtschaftsinformatik 2021 Proceedings

Track 10: Design, management and impact of

Al-based systems

\title{
Empirically Exploring the Cause-Effect Relationships of Al \\ Characteristics, Project Management Challenges, and \\ Organizational Change
}

\author{
Christian Engel* \\ University of St.Gallen, Switzerland \\ Philipp Ebel \\ University of St.Gallen, Switzerland \\ Benjamin van Giffen \\ University of St.Gallen, Switzerland
}

Follow this and additional works at: https://aisel.aisnet.org/wi2021

Engel*, Christian; Ebel, Philipp; and van Giffen, Benjamin, "Empirically Exploring the Cause-Effect

Relationships of Al Characteristics, Project Management Challenges, and Organizational Change" (2021).

Wirtschaftsinformatik 2021 Proceedings. 3.

https://aisel.aisnet.org/wi2021/QDesign/Track10/3

This material is brought to you by the Wirtschaftsinformatik at AIS Electronic Library (AISeL). It has been accepted for inclusion in Wirtschaftsinformatik 2021 Proceedings by an authorized administrator of AIS Electronic Library (AISeL). For more information, please contact elibrary@aisnet.org. 


\title{
Empirically Exploring the Cause-Effect Relationships of AI Characteristics, Project Management Challenges, and Organizational Change
}

\author{
Christian Engel ${ }^{1}$, Philipp Ebel ${ }^{1}$, Benjamin van Giffen ${ }^{1}$ \\ ${ }^{1}$ University of St.Gallen, Institute of Information Management, St.Gallen, Switzerland \\ \{christian.engel, philipp.ebel, benjamin.vangiffen\}@unisg.ch
}

\begin{abstract}
Artificial Intelligence (AI) provides organizations with vast opportunities of deploying AI for competitive advantage such as improving processes, and creating new or enriched products and services. However, the failure rate of projects on implementing AI in organizations is still high, and prevents organizations from fully seizing the potential that AI exhibits. To contribute to closing this gap, we seize the unique opportunity to gain insights from five organizational cases. In particular, we empirically investigate how the unique characteristics of $\mathrm{AI}$ - i.e. experimental character, context sensitivity, black box character, and learning requirements - induce challenges into project management, and how these challenges are addressed in organizational (sociotechnical) contexts. This shall provide researchers with an empirical and conceptual foundation for investigating the cause-effect relationships between the characteristics of AI, project management, and organizational change. Practitioners can benchmark their own practices against the insights to increase the success rates of future AI implementations.
\end{abstract}

Keywords: artificial intelligence, project management, case study, sociotechnical system

\section{Introduction}

Nowadays, Artificial Intelligence (AI) is a pervasive organizational and economic phenomenon [1]. These developments result from the persistent technological innovations in the areas of algorithmic capability, computing power, and data storage $[2,3]$. Thus, AI systems are increasingly applied in various usage contexts to both improve processes, and to enhance or create new products and services [4]. This potential has also been widely recognized in corporate practice. A recent survey among 3000 CIOs shows that the number of companies that has experimented with AI solutions has increased by 270 percent over the last four years [5]. However, it is estimated that throughout 2022, 85 percent of AI projects will fail to meet the intended targets and deliver erroneous outcomes [5], which shows a gap between understanding and successfully implementing AI solutions to leverage the potential that AI exhibits. 
In this paper, we focus on AI implementation projects as our unit of analysis, i.e. projects that develop and embed AI solutions in organizational (socio-technical) contexts. Particularly, we aim at contributing to paving the way for enriching knowledge on AI project management by seizing the unique opportunity to gain insights from multiple empirical cases. Thus, we pose the following research question:

How do AI projects induce socio-technical challenges into organizations and how can they be addressed by project management?

As research on AI project management practices is still nascent, this implies the need for empirical studies in the field of AI project management as the deduction of concepts from existing conceptualizations and theories is very limited, and (AI) projects are highly context-sensitive. Thus, over the duration of nine months, we engaged in five in-depth case studies with large corporations, which can be viewed as leading in their industries, in order to gain insights into the distinguishable challenges that implementing AI solutions induces into organizations and what the observed resolution approaches of the project management were to manage these challenges.

With this research, we aim to make contributions to both practice and research. First, this study aims at providing practitioners with unique empirical insights gained in realworld organizational settings, which shall create awareness for AI-specific challenges that lie ahead for organizations planning to implement AI solutions, and to present them with resolution approaches observable in corporate practice. Second, we aim to empirically enrich the scientific body of knowledge in the field of project management from a socio-technical perspective. This shall help researchers to better understand the interrelations between AI implementation projects as a focal unit of analysis and the components of socio-technical systems, i.e. the respective organizational context. Third, we aim to contribute to a conceptually sound and empirically rich research foundation on the cause-effect relationships between the characteristics of AI, the induced project management challenges, and the required changes in socio-technical systems. Overall, this shall help to make our unit of analysis, i.e. AI projects in specific, and $\mathrm{AI}$ as our phenomenon of interest in general more explainable and predictable.

\section{Conceptual Background}

We refer to a phenomenon-oriented definition of AI that characterizes it as machines performing the cognitive functions typically associated with human cognition, such as perceiving, interacting, reasoning, and learning [6]. We are aware that the term AI often raises wrong expectations regarding what machines are actually capable of doing, which still leads to hype-induced disappointments in many AI endeavors.

Thus, we narrow down the terminological scope of this paper seizing the common differentiations between "weak AI" that only pretends to think and "strong AI" that refers to a mind exhibiting mental states [7], as well as on a domain-oriented level to the categorization of narrow AI and Artificial General Intelligence (AGI) [8]. While narrow AI refers to an AI that is equally as good or better than a human in a specific domain of tasks, an AGI is posed to be equally as good or better than a human in any domain of tasks [8]. Consequently, when referring to AI in this paper, we mean weak and narrow AI rather than strong and general AI because the former has already proven 
its feasibility in the real world, and the latter is still more in the focus of philosophical debates and far away from being technically realized soon [9].

Against this backdrop, AI takes over certain degrees of cognition, which shall provide two types of AI outputs - decisions or solutions [2]. This is facilitated by advancements in computing power, algorithmic capabilities, and data storage [2, 3]. AI systems need to process large amounts of data and even Big Data in short time periods as they are approaching to match the intellectual performance of the human brain [10]. Thus, AI systems often require high levels of computing power. Second, advanced algorithms are used to replicate human cognition by learning from data and adapting to different contextual situations [11]. Thus, productive AI systems incorporate a learning capability to conduct decision making and problem solving [3]. Finally, the advancements in storing the data required for training, testing, commercializing, and maintaining AI solutions, facilitates the creation of a machine memory [12], which is regarded as the basis of intelligent systems [13].

Besides the technological facilitators, the following set of characteristics has been attributed to AI by research: experimental character (1), context sensitivity (2), black box character (3), and learning requirements (4) of AI. This set of characteristics is not claimed to be exhaustive but shall cover a representative share of the unique characteristics of AI, which we briefly summarize here:

(1) Experimental character refers to AI outcomes being non-deterministic but rather probabilistic [14].

(2) Context sensitivity refers to AI solutions being only as good as the data their context provides to reflect and predict the latter [15].

(3) Black box character refers to AI systems, especially in the field of deep learning, facing challenges in delivering explanations to humans on what happens between data input and AI output [16].

(4) Learning requirements refer to AI solutions constituting entities that just like humans need to learn and develop experience to eventually improve their performance over time [3].

This set of characteristics reflects that technological advancements on the one hand are necessary facilitators of AI solutions but lead to challenges that are induced into organizations intending to implement AI systems that have not existed before this digital era. For instance, the processes of generating AI models differ from programming traditional software applications. Software applications are based on code that runs predictably and deterministically on a computer, while AI models learn from processing data using machine learning [17]. We refer to the following notion of Machine Learning that grasps its underlying mechanism: "A computer program is said to learn from experience $\mathrm{E}$, with respect to some class of task $\mathrm{T}$ and performance measure $\mathrm{P}$, if its performance at tasks in $\mathrm{T}$, as measured by $\mathrm{P}$, improves with experience E" [18, p.2]. Generating machine learning models requires training [19], which refers 
to the process of extracting knowledge stored in the data by applying machine learning algorithms. This difference between programming software and training AI models has far reaching implications regarding the activities and practices in each type of project.

In this paper, we intend to empirically explore the cause-effect relationships of the set of AI characteristics described above, and how these induce project management challenges against the backdrop of the organizational context. Thus, we view project management challenges and the required organizational IS design and change through the lens of socio-technical systems (STS) theory that takes into account the interrelations of technology, task, structure, and actors [20,21].

\section{Method}

We opt for a qualitative, inductive reasoning from multiple cases $[1,22,23]$ to identify socio-technical challenges induced by AI technology. The case study method is appropriate for this type of research because the management of AI projects represents a context-sensitive phenomenon on which only little theory is available that acknowledges the technology-specific challenges. Here we describe the phases of case selection (1), data collection (2), and data analysis (3) of our research design.

For case selection (1), It was our particular goal to retrieve a discrete set of cases, i.e. AI projects that exhibit potential variation among another - be it variation in project goals or organizational contexts in distinct industries. Therefore, we chose five projects for case analysis, which stem from three distinct industries and exhibit three distinct project goals (see also Table 1). We purposefully selected the combination of project goal and industry in the cases to achieve a high level of variation in the ways that lead to project success according to Mill's method of agreement [24]. This is based on inducing insights from variation in independent case variables with the dependent case variable - i.e., "successfully implemented AI project" - being the same among the cases. To maintain comparability between the cases, we selected the organizational case environment of the particular tasks to be large corporations that already had to have deployed AI systems. Furthermore, we purposefully selected organizations that are leading in their particular industry to base the collection of observed resolution approaches on a revelatory foundation [23].

The process of data collection (2) took nine months, in which we investigated the five cases in relation to their particular organizational environment. We were offered with the unique opportunity of conducting case interviews on different hierarchical levels of the organizations. This helped us to develop a more comprehensive understanding of the different perspectives on AI projects in different socio-technical contexts. We approached the interviewees with semi-structured interviews conducted by three researchers from our research team. In doing so, we predefined an interview guideline that assured natural flow of conversations with the interviewees and allowed for variation in topics. Furthermore, this enabled us to adapt to the different levels of hierarchy, professional backgrounds and new themes that eventually may emerge in the interviews [23]. For each project, we additionally gathered case documents that were identified to be directly or indirectly related to the AI project (see also Table 1). 
Table 1. Case Information

\begin{tabular}{|c|c|c|c|}
\hline $\begin{array}{l}\text { Project / } \\
\text { (Industry) }\end{array}$ & AI Project Goal & $\begin{array}{c}\text { Interviewees } \\
\text { (Duration of Interviews) }\end{array}$ & Case Data \\
\hline $\begin{array}{l}\text { Alpha } \\
\text { (Telco) }\end{array}$ & $\begin{array}{l}\text { Classification and Routing } \\
\text { of Incoming Client Emails }\end{array}$ & $\begin{array}{c}\text { Head of Capability } \\
\text { Management (30 min.), } \\
\text { Project Owner from Business } \\
\text { (60 min.), Project Manager } \\
\text { (60 min.) }\end{array}$ & \multirow{5}{*}{$\begin{array}{l}\text { Interviews: } \\
\text { Transcripts } \\
\text { Internal files: } \\
\text { Project } \\
\text { document- } \\
\text { ations and } \\
\text { presentations } \\
\text { Public } \\
\text { Sources: News } \\
\text { articles, } \\
\text { company and } \\
\text { project } \\
\text { websites, and } \\
\text { business } \\
\text { reports }\end{array}$} \\
\hline $\begin{array}{c}\text { Beta } \\
\text { (Banking) }\end{array}$ & $\begin{array}{l}\text { Translation of Financial } \\
\text { Documents from Italian } \\
\text { and French to German }\end{array}$ & $\begin{array}{c}\text { Chief Information Officer } \\
\text { (40 min.), Project Manager } \\
\text { (120 min.) }\end{array}$ & \\
\hline $\begin{array}{c}\text { Gamma } \\
\text { (Manu- } \\
\text { facturing) }\end{array}$ & $\begin{array}{c}\text { Price Setting for } \\
\text { Individualized Technical } \\
\text { Offerings }\end{array}$ & $\begin{array}{c}\text { Chief Information Officer } \\
\text { (30 min.), Project Manager } \\
\text { (80 min.) }\end{array}$ & \\
\hline $\begin{array}{c}\text { Delta } \\
\text { (Banking) }\end{array}$ & $\begin{array}{l}\text { Classification, Routing } \\
\text { and Resolution of Internal } \\
\text { Incident Tickets }\end{array}$ & \begin{tabular}{|c|} 
Head of Data and \\
Analytics (40 min.), Head of \\
Platform Strategy (35 min.), \\
Project Manager (120 min.)
\end{tabular} & \\
\hline $\begin{array}{l}\text { Epsilon } \\
\text { (Manu- } \\
\text { facturing) }\end{array}$ & $\begin{array}{l}\text { AI-based classification, } \\
\text { routing and resolution of } \\
\text { internal incident tickets }\end{array}$ & \begin{tabular}{|c|} 
Vice President IT \\
Innovation (50 min.), Project \\
Manager (50 min.)
\end{tabular} & \\
\hline
\end{tabular}

The data analysis (3) was carried out on the foundation of the interview transcripts and case documents. Respectively, three researchers extracted data from the material and conducted open, axial, and selective coding iterations [25]. After an open coding iteration of the documents, the coders assigned relationships among the open codes (axial coding) in a second iteration. Subsequently, we purposefully defined the core variables for selective coding to be "challenges of AI projects" and "resolution approaches", which affect the dependent case variable "successfully implemented AI project". Three coders conducted the selective coding iteration against the backdrop of the conceptual background established for this research. After each coding iteration, the particular coding results were validated in research discussions among the three researchers [25]. This helped to identify a set of specific AI-induced project management challenges and to relate them to the particular socio-technical components of the organizational environments of the projects. Because the selected combinations of the goals and organizational contexts of the AI projects lead to a decent level of variation in the analyzed cases, the AI project management challenges can be induced on a broad conceptual basis [23]. 


\section{$4 \quad$ Results}

Figure 1 provides an overview of the identified set of specific challenges of planning, developing, and embedding AI solutions in organizations, and emphasizes the AIspecific root-causes of each of these challenges along with the observed socio-technical changes that could be observed in the cases. The AI-specific root-causes refer to the distinguishable characteristics of $\mathrm{AI}$ that were established in the conceptual background of this work, i.e., experimental character, the context sensitivity, the black box character, and the learning requirements of AI. Overall, this shall provide a first empirical basis for investigating the cause-effect relationships of AI characteristics, project management and organizational change.

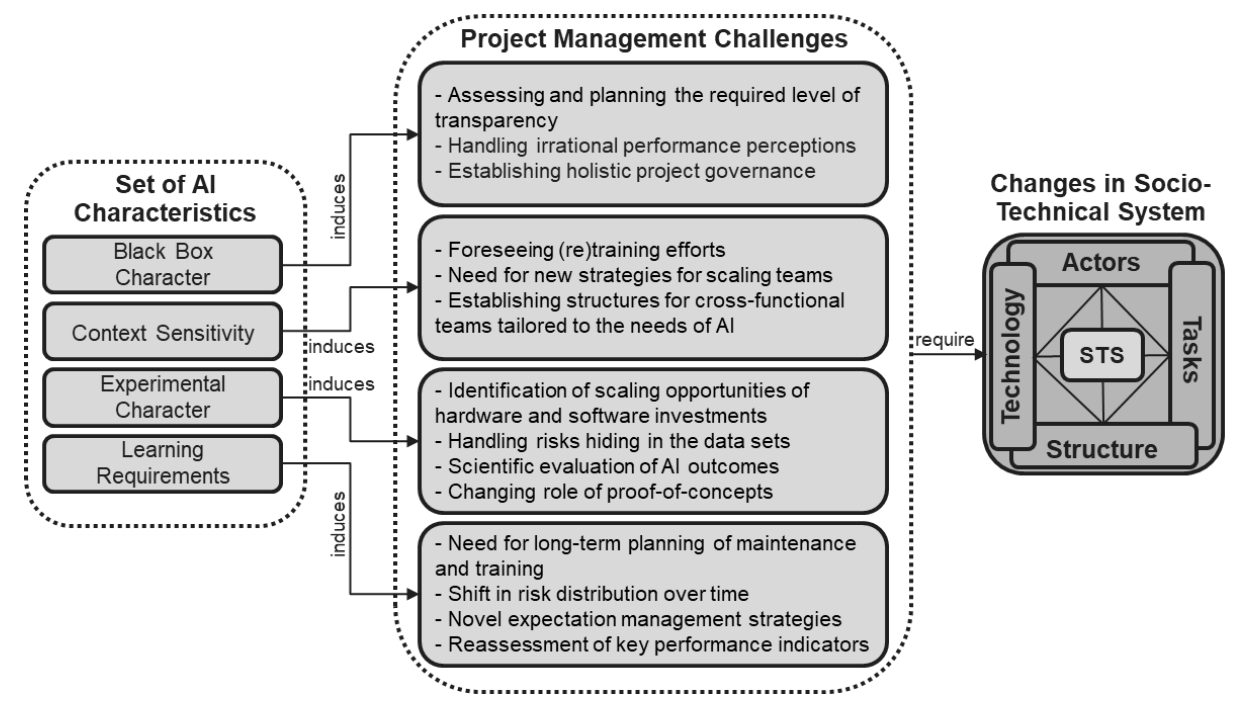

Figure 1. Proposed Cause-Effect-Relationships of AI Characteristics, Project Management Challenges, and Changes in the Socio-Technical System (STS)

\subsection{Black Box Character: Induced Challenges and Changes in STS}

First, the black box character of AI challenges project management to assess the required level of transparency of certain use cases, which requires change in the STS component of technology: The black box character that AI models often exhibit have led to the rise of the research on "explainable AI" that investigates the tradeoff between performance and transparency of models [26]. For project management, this means that project managers need to be aware that some use cases impose restrictions due to inherent transparency requirements rooted in the required auditability of processes. Furthermore, project management needs to control for transparency and hold developers accountable when introducing AI systems - for instance, by implementing rationalization into the system, i.e. the AI verbalizes its functioning [27]. 
"We use the AI itself to communicate more to the end user. For instance, in this project it is messaged at the beginning of the conversation [...]: 'Hey, now I currently I cannot handle orders but I can do this and that. '” - Project manager (Epsilon)

For example, in the case of the AI-based incident management system in the manufacturing context (project Epsilon). The system uses a chatbot as an interface that, after running testing iterations with users, had to be adapted to verbalize its actions and describe what it is capable of doing and what not. Project managers should foresee situations in which the black box characteristics of AI need to be mitigated, e.g. by further disclosing which sources are used, and what reasoning leads to AI decisions.

Second, the black box characteristics of AI can lead to an irrational performance perception, which requires change in the STS component of actors: As AI systems are non-linear systems [17], AI outcomes do not follow tree-like "if-then" structures, which causes dissonance between human decision heuristics and the respective AI output. This challenge is multiplied by the inherent bounded rationality of humans [28]. I.e., even though, AI may lead to objectively better results measured in numbers, people might still not be able to perceive this success due to the black box character of AI, as cases where AI makes mistakes might be easy cases for people observing these mistakes, and vice versa. For instance, in project Epsilon we observed an AI solution that correctly performed a certain task in 80 percent of the cases while a human actor correctly performed the task in 70 percent of the cases. However, actors looking into the 20 percent of cases that are incorrectly performed by the AI systems, might perceive these cases to be easy and obvious cases to handle. In the analyzed case, this led to people questioning the AI project outcomes as it is not transparent to them why the AI models are not able to cope with these "easy" and obvious cases. This can lead to a twist in actors' perception, even though the AI performs better than the human actors.

"You really need to make people understand what AI is, what is within this black box, so that they see for themselves that it is not purely one to one rules that are applied but it is much more." - Project manager (Epsilon)

To cope with the bounded rationality of humans, throughout the cases, the interviewees emphasized the necessity to constantly make people understand what AI is in general, and what hides within AI as a black box to make them understand that AI moves beyond pure one to one rules. For instance, in project Epsilon, collecting and communicating reference cases was observed to be a means of mitigating this challenge: These reference cases should be of high impact to show what the potential behind $\mathrm{AI}$ is. In that, it is important to constantly create awareness through various communication channels (face-to-face, and indirect), to repeat the message but also to showcase what is improving in the systems. Another concrete measure observed in the cases is to early integrate the end users of the systems in ideation and development phases. For instance, design thinking workshops were conducted before the actual kickoff of project Epsilon to prototype ideas and to early reach out to the end users.

Finally, the black box characteristics of AI make holistic project governance inevitable, which requires change in the STS component of structure: Due to the black box characteristics of AI - these vary in their intensity among the distinct algorithms it is hard to trace back a certain model output to certain input variables, which imposes 
the necessity on project management to establish particular governance mechanisms. Project management needs to consider ethical and cultural aspects of governing AI projects. Against the backdrop of recent scandals about racist, gender-discriminating AI solutions that made international news [29], these AI-specific governance mechanisms become of utmost importance for preventing AI projects from causing large-scale harm to the organization and its environment. Especially, when outsourcing AI development, these governance mechanisms lead to project management challenges as not everything is observable between a principal and an agent [30].

"Our company has a general contract with Microsoft and an agreement where most of these governance topics are covered." - Project manager (Epsilon)

In the case of AI-based incident management (project Epsilon) in the manufacturing industry, we could observe that these challenges were largely approached by establishing general contracts with suppliers on data safety and ethical governance, which lowered both transaction costs and project risks.

\subsection{Context Sensitivity: Induced Challenges and Changes in STS}

The context sensitivity of AI requires to foresee (re)training efforts, which requires change in the STS component of technology: Since the performance of AI systems is dependent on the context, it is necessary to account for a change in the environment by early planning the retraining of AI models [31]. For instance, in the case of determining the price level of complex technical offerings (project Gamma), changes in price driving parameters such as prices of natural resources, or changes in laws affecting legal requirements of offerings, e.g. regarding safety, impact whether an AI model is performant over time or not. Also, in project Delta, the models have to be up to date to work in the long run, i.e., taking into account changes in types of incoming incidents.

"[It] depends on what is the quantity of tickets that we get. That is where we need to sit down and discuss in terms of how we do that retraining of the model and the redeployment of the model itself." - Project manager (Delta)

Thus, the project managers early conducted assessments of environmental changes to adequately plan data cleaning and relabeling as part of their sprints based on the timeliness requirements of the data, i.e. planning the intervals of how often the models need to be retrained, making sure that enough of the right data in the right quality and accessibility is available at these points in time [32].

Furthermore, the context sensitivity of AI requires new strategies for scaling respective teams, which requires change in the STS component of actors: For scaling AI solutions across a company, distributed computing of the entire model [33], alignment of the distinct data sets as well as redundancies of data sets for individual computing are required [34]. In that, project managers face the challenge to plan and prepare managerial decision making on which parts of the core AI team, i.e., data scientists, data engineers, subject matter experts, product owners need to be scaled. 
"[...] The data engineering team can scale because they are the ones who manage it across different servers, or put it into the cloud but from a [...] modelling perspective I do not see a need to scale as we want to scale the usage." - Project manager (Delta)

From a data science and model creation perspective, the interviewed project managers did not face a necessity to increase the team size as the machine learning models did not vary heavily. The contrary was the case from a data engineering perspective as there is the challenge to handle the effort for gathering, cleaning, structuring, and storing the data from multiple silos within the organization as the data requirements across the organization can vary. Scaling this designated group of the core team needs to be planned in advance, efforts need to be estimated, and team integration of this particular group has to be managed [35].

Finally, the context sensitivity of AI requires to establish structures for crossfunctional teams, which requires change in the STS component of structure: Successful AI solutions require both domain expertise, and data science and engineering expertise. This means that business and IT departments need to establish structures to be able to closely collaborate in cross-functional teams as each AI problem is different depending on the organizational context [36]. In that, project managers face the challenge to establish these project structures and harmonize them with organizational structures to reach a close collaboration between business and IT departments to account for the context sensitivity of AI.

"The [external] team consists of computer experts, data experts and business experts. [Internally], there was a data expert, a salesman from the field [...], and I was there as a link." - Project manager (Gamma)

We observed in the cases that within the organizations semi-open project onboarding events are conducted with self-selection mechanisms. Furthermore, it became evident during the cases that the context sensitivity of AI requires to establish clear product ownership in the respective business departments that are trying to solve a business problem, as otherwise a continued use of the AI system beyond the project is unlikely.

\subsection{Experimental Character: Induced Challenges and Changes in STS}

In this vein, we could induce from the cases that the experimental character of AI implies the need to proactively search for scaling opportunities if significant hardware and software investments are necessary, which requires change in the STS component of technology: As of now, organizations have started investing in AI technologies but are vigilant and attentive regarding these investment decisions, due to the perceived newness of AI [37]. This is reflected in the size of lighthouse projects being small- and medium-sized in order to serve as a seed of AI that is planted into the organization. For instance, in the case of machine translation (project Beta), a flexible cloud solution could not be seized at the moment, due to the sensitivity of data related to the financial documents that should be translated. Thus, an on-premise server had to be sourced.

"The GPU server was expensive, but we would have found a use for it somewhere else in the company. The business case for the internal GPU server works and thus speaks positively for the GPU server internally." - Project manager (Beta) 
In project Beta, project management faced the need to start with a small but measurably successful use case before moving towards scaling the project scope. To justify investments in a GPU server, project management proactively searched for scaling opportunities to other use cases before respective investment decision rounds were triggered with line managers. As a consequence, investments in a GPU server were made in the course of the project with respect to extending it to further use cases, as the AI system for machine translation only uses a small share of the computing capacity of the GPU server.

Moreover, the experimental character of AI leads to a high level of project risks hiding in in the data, which requires change in the STS component of task: In the cases, we could find empirical evidence for a large portion of project risk hiding in the data sets that are required for training and testing of models. For instance, while training their AI-based customer incident management system (project Alpha), the project manager of the telecommunication provider observed that, when labelling more data and feeding more data into the AI model, the model's performance became worse, which led to a fair share of initial confusion. However, it could be identified that this was rooted in flawed data sets, which were caused by mistakes committed by the subject matter experts when labelling the data. In a similar vein, the project manager of project Delta summarized the role of data in the respective AI project on incident management.

"[...] I have always been aware of [...] the risk of having the data quality and interpretation of the data, and subject matter knowledge." - Project manager (Delta)

Thus, project management introduced data quality assurance mechanisms into the AI projects and the organization, such as peer data reviews, data stage-gates, and automated data screenings [38].

Furthermore, the experimental character of AI requires a scientific evaluation of results, which requires change in the STS component of task: As AI systems operate probabilistically, this requires to evaluate the final AI solution or to test minimum viable products (MVPs) in a scientific manner that is reproducible, rigorous, and robust [39]. For instance, in the case of translating financial documents (project Beta), the bank set the goal to deliver results with higher translation quality than a global market leader of AI-based translation solutions in at least 60 percent of the cases.

"The team of four translators then spent a maximum of four hours assessing the equivalence of the [benchmark tool] outputs and the in-house tool outputs, from which the quality score was calculated." - Project manager (Beta)

Several individual sentences in the source language with the respective translation from the newly developed AI-based machine translation were compiled for the translators in one document. The translators then had time to comment on the output, e.g. on the style used, spelling, language usage, etc. This was done with 400 test translations. These were generated from the benchmark tool of the global market leader and the developed tool. There were four translators per language, two from the bank's team and two external translators whom the supplier of the AI solution could select. This four-person team of translators then evaluated the equivalence of the benchmark tool outputs and the in-house tool outputs, and a quality indicator was calculated. Test dummies were also built in to test the attention of the translators. 
Finally, the experimental character of AI initiatives changes the role of proof-ofconcepts (PoCs), which requires change in the STS component of structure: Throughout the cases we could observe that the role of proof-of-concepts (PoCs) changes and thus risk is increasingly transferred from the supplier to the buyer. This is especially the case when the AI solution is sourced from outside of the company and project management is more concerned with embedding the AI solution in the sociotechnical context. This imposes structure-related challenges on project management as this shift in risk needs to be mitigated to be bearable by the accountable stakeholders. For example, in the case of machine translation of financial documents (project Beta), a PoC was not possible because two neural networks had to be extensively trained.

"We made a bonus-malus clause as the classic POC that you always strive for when creating MVPs is often not possible. You have to do it just right, because you can't train a [neural] net with a few data points." - Chief Information Officer (Beta)

For this reason, in project Beta, a bonus-malus clause tailored to the expected AI performance was negotiated in the supplier contracts in line with value-based pricing. Translating and mediating between sales and purchasing department, external developers, internal solution integrators, and C-level management was part of the project management effort in establishing this bonus-malus clause.

\subsection{Learning Requirements: Induced Challenges and Changes in STS}

First, the learning requirements of $\mathrm{AI}$ induce the need for long-term planning of maintenance, which requires change in the STS component of technology: Like an employee, AI systems face the need to learn, and in specific case to even conduct lifelong learning. This is due to data value being time-dependent [40] and requires project managers to move beyond the set time frame of a project when conducting planning, and to apply a long-term view on how AI systems need to be maintained to account for long-term changes such as changing user perceptions, values, and culture. In the case of translating financial documents (project Beta), project managers faced the inherent risk that the financial jargon evolves over time [41], which needs to be taken into account when intending to deliver a performant system that is built for the long-run.

"It is not like we develop a model today and then it runs for the next ten years. We have to retrain it like a human being. We learn every day, so it also needs to learn on a daily basis. That is the pipeline that we plan to build when we actually go in 'production'." - Project manager (Delta)

Interviewed project managers emphasized the need to become aware that a model that is developed today does not run successfully in the long-run without retraining it just like a human being needs to regularly be trained to stay up-to-date. Thus, in planning the project, project managers went beyond the time frame of the actual implementation of the AI system to establish a continuous retraining and testing pipeline. E.g. in project Delta, project managers planned to source customer incident data directly via APIs to feed the AI models, even long after the actual project transferred to a productive state. The AI models are then to be continuously evaluated with the new data sets, and then eventually have to be adapted, extended, and improved. 
Second, the learning requirements of AI lead to a shift in risk distribution over time, which requires change in the STS component of task: The learning requirements of AI lead to the required data volume, quality, and effort of model tuning needed for PoCs being significantly substantial in AI projects. Especially, the data cleaning steps are very time consuming if the initial quality of the data sets is low. In the analyzed cases, we could observe that due to a proliferation of open source AI models, model creation did not induce as much project effort as the data preparation steps. This implies that once feasibility is proven in a PoC, a major share of the project is already finished, which leads to a shift in risk distribution over the time period of AI projects, which needs to be mitigated by project management. To cope with this challenge, throughout the observed cases, project management approached the PoCs in a hypotheses-driven manner for certain building block of the solution by creating a first version of the solution (minimum viable product) and testing it with a small number of end users.

"We gradually processed more and more incoming emails fully automatically until 100 percent of the requests were processed automatically." - Project manager (Alpha)

After rolling it out in a pilot over the business units, we could observe that throughut the cases user feedback was gathered, then it was rolled out again to a constantly increasing number of users in a productive environment.

Furthermore, the learning requirements of AI imposes new challenges on stakeholder communication and expectation management, which requires change in the STS component of actors: This actor-related challenge is multiplied by the AI hype that is prevalent nowadays [42] and thus imposes challenges on managing expectations towards AI projects. AI solutions constitute learning systems that improve their performance over certain time spans, and as stated earlier in some use cases even require life-long learning. Thus, project management faces the challenge of communicating this to all relevant stakeholders in order to establish understanding of and patience towards expected AI outcomes. In the case of AI-based classification, routing and resolution of internal incidents tickets (project Epsilon), project management thus developed a story around the project to transport the message that their AI system learns and evolves. However, the project management was faced with users expecting that everything works perfectly right after the implementation of the AI solution. This imposed significant communication efforts in terms of providing information on future advancements, in order to manage the expectations of the users.

"AI has to be trained. AI should be regarded as an intern, otherwise users will perceive it as not useful. One team cancelled an AI solution after two weeks because the AI was not able to resolve 100 percent of the issues." - Project manager (Epsilon)

In project Epsilon, besides announcements that were made to people via email, the AI itself was used to communicate to the end users what it is capable of doing now and what it will be capable of doing after a sufficient learning period and the metaphor of "AI as an intern" was established.

Finally, the learning requirements of AI demand a reassessment of key performance indicators, which requires change in the STS component of structure: During the case analysis, it became evident that traditional IT solution performance measurements such as up-time, number of capacity-relate incidents etc. as provided for instance by COBIT 
(Control Objectives of Information and related Technology) and ITIL (IT Infrastructure Library) need to be tracked for guaranteeing the general stability of the AI solution. However, this is more or less regarded as a hygiene factor but does not establish respective measurement structures for keeping track of the performance of the AI system from a value-oriented perspective [43]. For instance, in the case of AI-based classification, routing and resolution of internal incidents tickets (project Epsilon), the project management faced the challenge to rethink their KPIs, finally moving to a more human-oriented performance assessment that was then applied to the machine.

"What is different in AI projects is that you rather look at KPIs that are relevant for people performance than IT solution performance." - Project manager (Gamma)

To account for the differences that are induced by the learning requirements of AI, project management (project Epsilon) established a KPI system to assess the performance of the AI system based on KPIs that had been originally used to assess the performance of humans rather than IT solution performance. In this specific case, the project manager of project Epsilon had a set of performance KPIs for their human service desk agents in place such as time to provide the solution, first time resolution, and percentage of incorrectly assigned incidents. These were then deployed to assess the performance of the AI system, and combined with the traditional IT performance measures. This shows, how the learning requirements of $\mathrm{AI}$ led to a structure-related change in the components of a socio-technical system through inducing respective challenges on implementing AI projects in an organizational environment.

\section{Discussion and Contribution to Research and Practice}

This research is motivated by the goal to contribute to answering the question of how AI projects induce distinguishable socio-technical challenges into organizations, and how these challenges can be handled, specifically by project management.

We note here that in no way the empirically induced set of AI-specific sociotechnical challenges and resolutions can hold to be exhaustive. This is rooted in the nature of empirical work within nascent fields of research. Thus, we acknowledge the extendibility of our results and look forward to future research building up on and extending our empirical findings.

To facilitate this process, the presented insights shall pave the way towards a deeper understanding of implementing AI in organizational settings, and of the specific project management practices required to overcome the obstacles that are induced by the specific characteristics of AI, which lead to a gap between understanding and leveraging the potential of AI in organizations. We purposefully position this paper as a means to provide insights to practitioners and to trigger future research in this nascent field, which shall provide a conceptually valuable and empirically grounded starting point for investigating the following links:

First, in this paper we establish a link between the unique characteristics of AI - i.e. experimental character [14], context sensitivity [15], black box character [16], and learning requirements of AI [3] - and the particular challenges that could be observed in our five cases. This shall provide researchers with the means to build up on these 
links in order to investigate how the characteristics of AI pose root-causes of required organizational change, and to provide answers from IS research for managing the latter.

Second, we purposefully chose organizations that are leading in their particular industry to base the collection of observed resolution approaches on a revelatory foundation [23]. In that, the presented resolutions shall provide a first - of course neither generalizable nor exhaustive - set of pathways forward. We also acknowledge that the presented resolutions may not be optimal, but however serve the purpose of industry leading organizations in the respective project settings. Through this second link of observed challenges and resolution strategies, which have proven to be successful, practitioners from the realm of project management can seize these aggregated insights to guide future $\mathrm{AI}$ implementation endeavors, or benchmark their own existing approaches. On the other hand, researchers can contribute to finding new ways of optimizing the problem-solution links by either enhancing the approaches presented in this paper, establishing new connections between problem and solution space, or extending either of it.

Third, through linking the challenges and resolutions to the dimension of sociotechnical systems theory, we intend to embed AI project management practices into a larger organizational context $[20,21]$, which shall pinpoint the implications that studying project management as a unit of analysis has in the context of AI. For research, this shall make the phenomenon of AI in general, and AI project management in particular more graspable, in a manner of showcasing cause-effect-relationships that need to be taken into account when trying to comprehensively address AI implementation from a project management perspective. The proposed set of causeeffect relationships thus shall serve as an empirical foundation for a vivid scientific discourse and exchange to advance this nascent field. Furthermore, it shall serve as a potential starting point for developing or adapting (new) constructs, models, methods, and ultimately theory in the realm of managing AI projects and the respective organizational change. For practitioners, the proposed cause-effect relationships provide an overview of how AI makes new decisions, solutions, and actions in project management necessary, and how these can affect the distinct socio-technical areas within their organization. Thus, the findings of this study shall support practitioners in assessing the potential consequences of the decisions they make when implementing $\mathrm{AI}$ in their organization. This can lower the still high failure rates of these projects [5]. 


\section{References}

1. Bamberger, P.A.: AMD - Clarifying What We Are about and Where We Are Going. Acad. Manag. Discov. 4, 1-10 (2018).

2. von Krogh, G.: Artificial Intelligence in Organizations: New Opportunities for Phenomenon-Based Theorizing. ACM Discov. 4, 404-409 (2018).

3. Jordan, M.I., Mitchell, T.M.: Machine learning: Trends, perspectives, and prospects. Science (80-. ). 349, 255-260 (2015).

4. Davenport, T.H.: From analytics to artificial intelligence. J. Bus. Anal. 1, 73-80 (2018).

5. Rowsell-Jones, A., Howard, C.: CIO Survey: CIOs Have Awoken to the Importance of AI. (2019).

6. Rai, A., Constantinides, P., Sarker, S.: Editor's Comments: Next-Generation Digital Platforms: Toward Human-AI Hybrids. Manag. Inf. Syst. Q. 43, iii--ix (2019).

7. Searle, J.R.: Minds, brains, and programs. Behav. Brain Sci. 3, 417-457 (1980).

8. Gubrud, M.A.: Nanotechnology and international security. In: Fifth Foresight Conference on Molecular Nanotechnology (1997).

9. Kumar, K., Thakur, G.S.M.: Advanced Applications of Neural Networks and Artificial Intelligence: A Review. Int. J. Inf. Technol. Comput. Sci. 4, 57-68 (2012). https://doi.org/10.5815/ijitcs.2012.06.08.

10. Moravec, H.: When will computer hardware match the human brain? J. Evol. Technol. 1, 1-12 (1998).

11. Noor, A.K.: Potential of Cognitive Computing and Cognitive Systems. Open Eng. 5, 75-88 (2015).

12. O'Leary, D.E.: Artificial intelligence and big data. IEEE Intell. Syst. 28, 96-99 (2013).

13. Stanfill, C., Waltz, D.L.: Toward memory-based reasoning. Commun. ACM. 29, 12131228 (1986).

14. Amigoni, F., Schiaffonati, V.: Ethics for robots as experimental technologies: Pairing anticipation with exploration to evaluate the social impact of robotics. IEEE Robot. Autom. Mag. 25, 30-36 (2018).

15. Lieberman, H., Selker, T.: Out of context: Computer systems that adapt to, and learn from, context. IBM Syst. J. 39, 617-632 (2000).

16. Castelvecchi, D.: Can we open the black box of AI? Nature. 538, 20-23 (2016).

17. Wilamowski, B.M., Irwin, J.D.: Intelligent systems. CRC press (2018).

18. Mitchell, T.M.: Machine Learning. McGraw-Hill, New York (1997).

19. LeCun, Y., Bengio, Y., Hinton, G.: Deep learning. Nature. 521, 436-444 (2015).

20. Bostrom, R.P., Heinen, J.S.: MIS problems and failures: a socio-technical perspective, part I: the causes. MIS Q. 1, 17-32 (1977).

21. Lyytinen, K., Newman, M.: Explaining information systems change: a punctuated socio-technical change model. Eur. J. Inf. Syst. 17, 589-613 (2008).

22. Eisenhardt, K.M.: Building Theories from Case Study Research. Acad. Manag. Rev. 14, 532-550 (1989).

23. Yin, R.K.: Case Study Research: Design and Methods. Sage Publications (2013).

24. Savolainen, J.: The rationality of drawing big conclusions based on small samples: in defense of Mill's methods. Soc. forces. 72, 1217-1224 (1994).

25. Forman, J., Damschroder, L.: Qualitative Content Analysis. In: Jacoby, L. and Siminoff, L.A. (eds.) Empirical Methods for Bioethics: A Primer (Advances in Bioethics, Volume 11). pp. 39-62. Emerald (2007).

26. Theodorou, A., Wortham, R.H., Bryson, J.J.: Why is my robot behaving like that? Designing transparency for real time inspection of autonomous robots. AISB 2016. 6366 (2016).

27. Wortham, R.H., Theodorou, A., Bryson, J.J.: What does the robot think? Transparency as a fundamental design requirement for intelligent systems. In: Ijcai-2016 ethics for 
artificial intelligence workshop (2016).

28. Bettis, R.A., Hu, S.: Bounded rationality, heuristics, computational complexity, and artificial intelligence. In: Behavioral Strategy in Perspective. pp. 139-150. Emerald Publishing Limited (2018).

29. Zou, J., Schiebinger, L.: AI can be sexist and racist-it's time to make it fair, (2018).

30. Akerlof, G.A.: The market for "lemons": Quality uncertainty and the market mechanism. Q. J. Econ. 84, 488-500 (1978).

31. Brézillon, P.: Context in Artificial Intelligence: II. Key elements of contexts. Comput. Artif. Intell. 18, 425-446 (1999).

32. Wang, R.Y., Strong, D.M.: Beyond accuracy: What data quality means to data consumers. J. Manag. Inf. Syst. 12, 5-33 (1996).

33. Konar, A.: Artificial intelligence and soft computing: behavioral and cognitive modeling of the human brain. CRC press (2018).

34. Lawrence, N.D.: Data Science and Digital Systems: The 3Ds of Machine Learning Systems Design. arXiv Prepr. arXiv1903.11241. (2019).

35. Huebner, A., le Cessie, S., Schmidt, C.O., Vach, W.: A contemporary conceptual framework for initial data analysis. Obs Stud. 4, 171-192 (2018).

36. Sandkuhl, K.: Putting AI into Context-Method Support for the Introduction of Artificial Intelligence into Organizations. In: 2019 IEEE 21 st Conference on Business Informatics (CBI). pp. 157-164 (2019).

37. Brynjolfsson, E., Rock, D., Syverson, C.: Artificial intelligence and the modern productivity paradox: A clash of expectations and statistics. (2017).

38. Gao, J., Xie, C., Tao, C.: Big Data Validation and Quality Assurance--Issuses, Challenges, and Needs. In: 2016 IEEE symposium on service-oriented system engineering (SOSE). pp. 433-441 (2016).

39. Park, S.H., Han, K.: Methodologic guide for evaluating clinical performance and effect of artificial intelligence technology for medical diagnosis and prediction. Radiology. 286, 800-809 (2018).

40. Heinrich, B., Klier, M.: Assessing data currency—a probabilistic approach. J. Inf. Sci. 37, 86-100 (2011).

41. Christiansen, M.H., Kirby, S.: Language evolution: Consensus and controversies. Trends Cogn. Sci. 7, 300-307 (2003)

42. Hopgood, A.A.: Artificial intelligence: hype or reality? Computer (Long. Beach. Calif). 36, 24-28 (2003).

43. Barrett, D.G.T., Hill, F., Santoro, A., Morcos, A.S., Lillicrap, T.: Measuring abstract reasoning in neural networks. In: International Conference on Machine Learning. pp. $4477-4486$ (2018). 